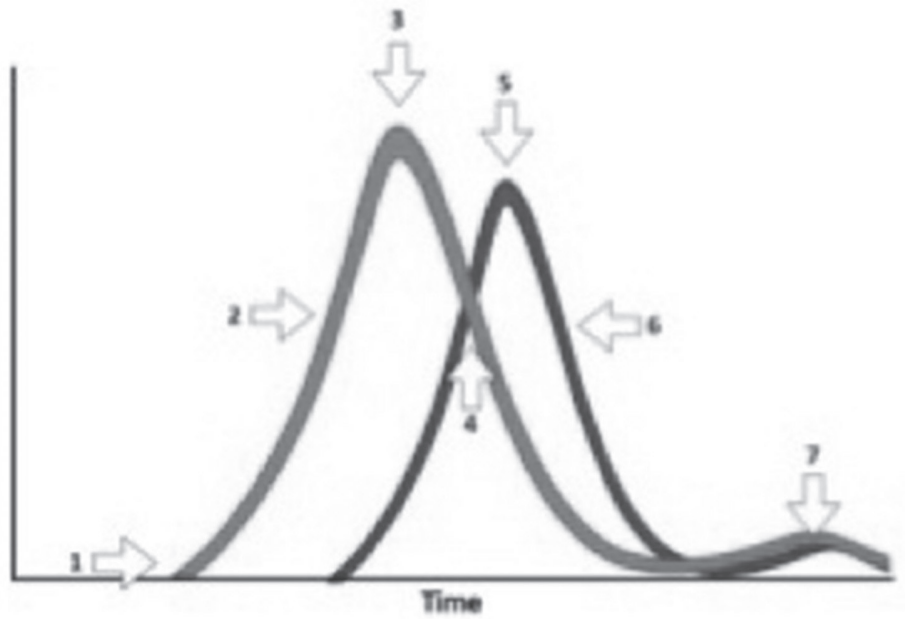

Abstract P-003 Figure 1 TAC curve; red = arterial input, blue = venous. 1: Baseline Arterial, 2: Prepeak, 3: Peak, 4: Notch, 5: Peak venous, 6: Venous downslope, 7: Venous eq.

\section{P-003 HYPOATTENTUATION ON CTA IMAGES EVALUATED FOR POTENTIAL ACUTE STROKE THROMBECTOMY: HOW TIMING AFFECTS CONSPICUITY}

C Lum, P Dave, R Thornhill, S Chakraborty, D Dowlatshahi. University of Ottawa-Ottawa Hospital Research Institute, Ottawa, ON, Canada

\subsection{6/neurintsurg-2016-012589.45}

Introduction CTA is used to triage acute stroke patients for potential thrombectomy. Single, multiphase ${ }^{1}$ or dynamic ${ }^{2}$ CTA may demonstrate areas of parenchymal hypoattenuation on source images(CTASI). These areas may represent either delayed arrival of contrast or could be ischemic/infarcted tissue. The area can be variable depending on the arrival of contrast on the time-tissue attenuation curve (TAC). Areas of hypoattenuation distal to an occlusion are perceived because of differences in contrast compared to normally-perfused areas and can be objectively measured. Patients with significant hypoattenuation may be potentially excluded from thrombectomy.

We evaluated conspicuity of hypoattenuation on dynamic multiphase CTASI. Specifically, we sought to determine if there is a specific time point on the TAC curve which optimizes conspicuity.

Materials and methods We retrospectively identified 45 consecutive large vessel strokes between 2012-14 who had dynamic CTA and CTP.

We identified ischemic areas from areas of low CBV. An ROI was drawn on the corresponding CTASI and in normally-perfused tissue.

We evaluated CTASI in $7 / 18$ points along the TAC (Figure 1). We calculated absolute and relative change in attenuation between potentially ischemic and normally-perfused tissue.

Differences in conspicuity were evaluated using a Friedman test with Bonferroni correction.

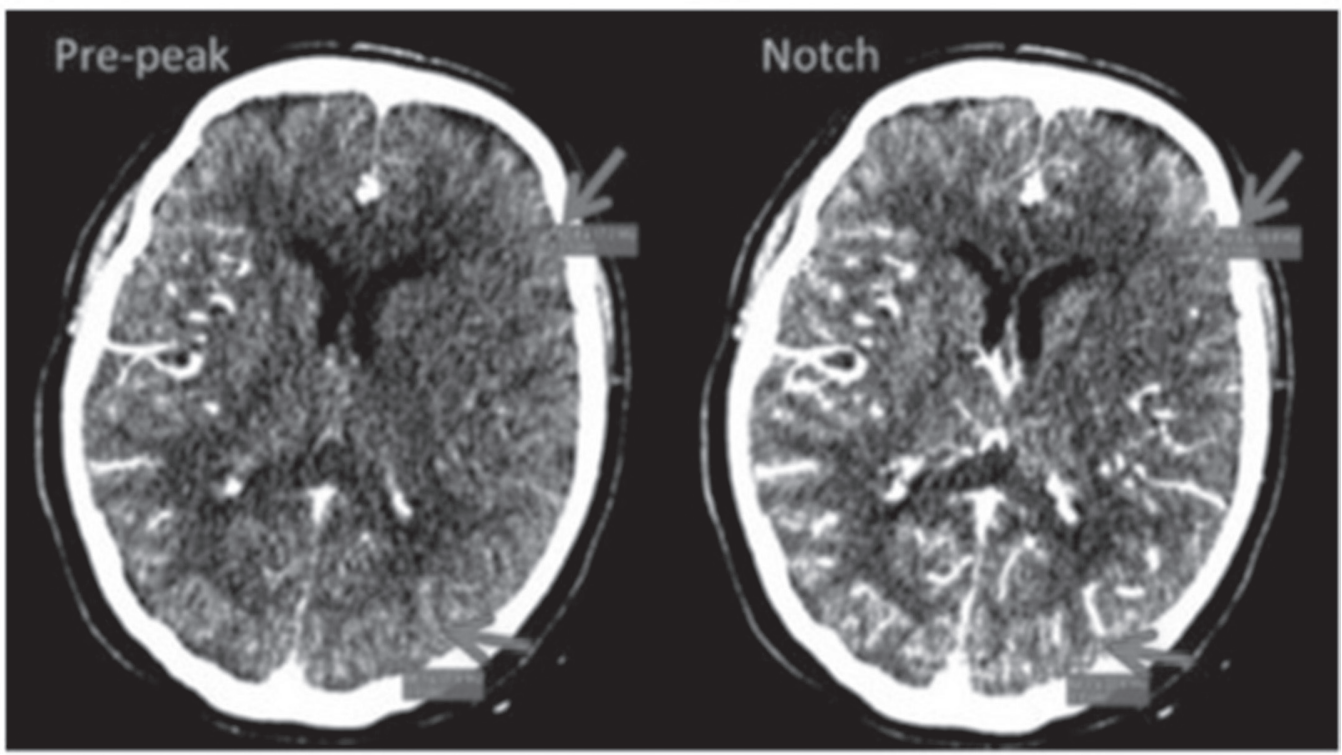

Abstract P-003 Figure 2 Identical slices at 2 different (pre-peak \& notch) phases of CTASI. The conspicuity of the ischemic area in the left frontal lobe (blue arrows) compared to normally-perfused tissue in the left parietal lobe (red arrows) is greater on the notch phase 
Results There were 22 males/23 females, age 69, \pm 16 , median NIHSS 10.6. All occlusions were in the anterior circulation; $64 \%$ on the left.

There was a significant effect of phase on the TAC for both absolute and relative conspicuity of ischemic (Figure 2) vs normally-perfused areas $(\mathrm{P}<0.00001)$.

The median absolute and relative conspicuity of ischemic:normally-perfused tissue was greatest at the peak arterial $(8.6 \mathrm{HU}$, 1.15), notch (9.4 HU, 1.17) and peak venous phases (7 HU, 1.13) vs other portions of the TAC (2.5-3.3 HU, 1.04-1.09).

Conclusion The conspicuity of ischemic areas distal to a large artery occlusion in acute stroke is dependent on the phase of contrast arrival on time-resolved dynamic CTASI. The greatest conspicuity of areas of hypoattenuation between ischemic and normally perfused tissue is in the middle of the TAC.

\section{REFERENCES}

1 Radiology 2015 May;275(2):510-20.

2 Stroke 2014 Sep;45(9):2683-28.

Disclosures C. Lum: None. P. Dave: None. R. Thornhill: None. S. Chakraborty: None. D. Dowlatshahi: None.

\section{P-004 PUBLISHING TITLE: INCIDENCE OF LARGE VESSEL OCCLUSIONS AMONGST ALL HOSPITAL DISCHARGES FOR ACUTE ISCHEMIC STROKE - ESTIMATING A THROMBECTOMY ELIGIBLE POPULATION}

\begin{abstract}
${ }^{1} \mathrm{~A}$ Rai, ${ }^{2} \mathrm{P}$ Link, ${ }^{3} \mathrm{~S}$ Boo, ${ }^{3} \mathrm{~J}$ Domico, ${ }^{3} \mathrm{~N}$ Lucke-Wold, ${ }^{4} \mathrm{~A}$ Tarabishy, ${ }^{3} \mathrm{~J}$ Carpenter. ${ }^{1}$ Interventional Neuroradiology, Ansaar Rai, Morgantown, WV; ${ }^{2}$ Stryker Neurovascular, Fremont, $C A ;{ }^{3}$ Interventional Neuroradiology, West Virginia University, Morgantown, WV ${ }^{4}$ Neuroradiology, West Virginia University, Morgantown, WV
\end{abstract}

\subsection{6/neurintsurg-2016-012589.46}

Background Endovascular therapy has been endorsed for emergent large vessel occlusions (ELVO). However the incidence of ELVO is ill defined and speculative. A methodical estimate of these patients is important for developing systems of care, resource allocation and market projections.

Objective To determine the national burden of ELVO by extrapolating these rates from a tertiary rural health system that captures over $85 \%$ of all ischemic strokes within its large rural catchment area to the national inpatient sample (NIS) database.

Methodology All hospital discharges with a primary or secondary diagnosis of AIS (ICD-9 433.xx, 434.xx, 435.xx) over a 3 year period in a rural tertiary health system in the "strokebelt" were evaluated for ELVO (ICA-T, MCA, BA) based on admission CT or MR angiography. For anterior circulation strokes, an ASPECTS $\geq 6$ was considered favorable for thrombectomy. The same ICD-9 codes were utilized to query the NIS-database to determine all AIS hospital discharges nationally and the ELVO rates were estimated based on the results from our catchment population.

Results There were 2757 AIS hospital discharges over a 3 year period (March 2012 to March 2015) based on the selected ICD-9 codes. An ELVO was present in 324 (11.8\%) of these patients. Out of these, M1-occlusion was present in 232 (71.6\%), ICA-T in $37(11.4 \%)$ and BA in $55(17 \%)$ patients. The majority of ELVO patients ( $\mathrm{n}=174,53.7 \%$ ) presented within 6 hours of last seen normal (LSN). Of the remaining $150(46.3 \%)$ patients: $45(13.6 \%)$ presented at 612 hours, $60(18.5 \%)$ at $12-24$ hours and $27(8.3 \%)$ at greater than 24 hours of LSN. There were 19 (5.9\%) patients with unknown LSN. For anterior circulation ( $\mathrm{n}=269,83 \%)$ an ASPECTS $\geq 6$ was observed in 118 of 150 (78.7\%) patient presenting within 6 hours of LSN and in 66 of 1119 (55.5\%) patients presenting after 6 hours of LSN ( $p<0.0001)$. The same ICD-9 codes for the NIS-database returned 1,135,030 AIS discharges nationally for 2013. Applying the statistics from our population to the NIS-database yields 133,388 large vessel strokes nationally $(95,512 \mathrm{M} 1,15,233 \mathrm{ICA}-\mathrm{T}$ and $22,643 \mathrm{BA})$. Of these, 71,634 patients are estimated to present within 6 hours and 61,754 after 6 hours of LSN. There could be 48,579 patients within 6 hours and 27,172 patients after 6 hours with an ASPECTS $\geq 6$ resulting in a total of 75,751 potential thrombectomy eligible patients in the anterior circulation.

Conclusion Almost $12 \%$ of the patients in our large rural sample of AIS hospital discharges presented with an ELVO. This translated to 133,388 patients when extrapolated to the NIS-database for 2013. An estimated 48,579 patients within 6 hours and 27,172 patients after 6 hours could be thrombectomy eligible based on ASPECTS $\geq 6$. An additional 22,643 patients were estimated to have a basilar occlusion. These are estimates based on a stroke center within a healthcare system serving a large rural population and probably represent the upper limit since the area under study has one of the highest ischemic stroke rates in the country. These numbers can also be subject to regional variations but nonetheless serve as a starting point for estimating the ELVO stroke burden and for planning systems of care.

Disclosures A. Rai: 1; C; Stryker Neurovascular. 2; C; Stryker Neurovascular. P. Link: None. S. Boo: None. J. Domico: None. N. Lucke-Wold: None. A. Tarabishy: None. J. Carpenter: None.

\section{P-005 AFTER MR SELECTION, FINAL INFARCT VOLUME IS INDEPENDENT OF TIME TO THROMBECTOMY FOR ANTERIOR LARGE VESSEL OCCLUSION STROKE}

B Cristiano, M Pond, S Basu, U Oyoyo, J Jacobson. Neuroradiology, Loma Linda University Hospital, Loma Linda, CA

\subsection{6/neurintsurg-2016-012589.47}

Purpose With thrombectomy for anterior circulation large vessel occlusion (ACLVO) stroke, time to recanalization is considered important, but collateral status may be a greater driver of outcome than time. We tested the hypothesis that patients with ACLVO stroke who present with a small core infarct on DWI would show similar good outcomes after thrombectomy, regardless of time from onset.

Materials and methods A cohort of 49 patients treated with thrombectomy after MR selection for ACLVO stroke from 11/1/2012 until 5/15/2015 was retrospectively reviewed. Patients were selected for thrombectomy based on DWI screening with presentation core volume $\leq 100$ age considered favorable. Patients were divided into early $(n=24)$ or extended $(n=25)$ treatment groups, with intention to treat $\leq 6$ hours $=$ early, and comparisons made with final infarct volume the primary outcome.

Results Baseline characteristics were similar (early versus extended), including admission NIHSSS (IQR 13-19 versus 11-18). Recanalization $\geq$ TICI2B was $79 \%$ and $83 \%$ respectively. There was no significant difference in median final infarct volume $(16 \mathrm{~mL}$ versus $22 \mathrm{~mL}$, estimated difference 